\title{
A Quantum Chemistry Approach to Possible Sulfur Adsorbate Structures on the Basal Plane of Graphite Clusters ${ }^{\dagger}$
}

\author{
J. L. Vicente, E. E. Mola, G. Appignanessi, J. L. Zubimendi, L. Vázquez, \\ R. C. Salvarezza, and A. J. Arvia* \\ Instituto de Investigaciones Fisicoquímicas Teóricas y Aplicadas (INIFTA), Sucursal 4, \\ Casilla de Correo 16, (1900) La Plata, Argentina
}

Received August 31, 1994. In Final Form: February 23, $1995^{\otimes}$

\begin{abstract}
A quantum chemistry study for possible sulfur adsorbate structures on the basal plane of graphite clusters has been made using the MNDO method. The potential energy curves showed the possible formation of $\mathrm{S}$ atoms, sulfur dimer and trimer adsorbates on top and bridge substrate positions, whereas neither sulfur atom, dimer, nor trimer adsorption binding to hollow position could be formed. Results gave support to a recently reported interpretation for sulfur atom adsorption on the basal plane of graphite based upon scanning tunneling microscopy data.
\end{abstract}

\section{Introduction}

The adsorption of sulfur on metal surfaces of welldefined fcc and bcc single crystals produces a number of sulfur overlayer structures ${ }^{1}$ which can be distinguished by scanning tunneling microscopy (STM) ${ }^{2-8}$ Several of these structures coexist at fractional monolayer (ML) coverages and their relative contributions depend on the degree of substrate surface coverage by sulfur atoms. In all these cases, sulfur adsorption takes place at hollow lattice substrate sites. ${ }^{1-8}$ Furthermore, for sulfur overlayer growth at the submonolayer level, a marked influence of the substrate on the sulfur-sulfur distance was observed. ${ }^{4}$

STM images (Figure 1A) resulting from sulfur adsorption on $\mathrm{C}(0001)$, at the submonolayer level, ${ }^{9}$ show a hexagonal underlying lattice with a side distance $d_{\mathrm{C}-\mathrm{C}} \cong$ $0.14 \mathrm{~nm}$. This $\mathrm{C}-\mathrm{C}$ bond distance corresponds to the honeycomb lattice of $C(0001)$ which can be observed under high resolution. In addition, bright spots related to sulfur atoms are observed. From these STM images which are extensively described elsewhere, ${ }^{9}$ it is clear that locations of sulfur atoms on the substrate do not coincide with hollow sites of the hexagons, but it is difficult to decide whether sulfur atom adsorption occurs at bridge or top positions. It should be noted that the adsorption of several gases

${ }^{\dagger}$ Presented at the symposium on Advances in the Measurement and Modeling of Surface Phenomena, San Luis, Argentina, August 24-30, 1994.

₹ Visiting Professor from the Instituto de Ciencia de Materiales, CSIC, Universidad Autónoma de Madrid, C-XII, 28049 Madrid, Spain.

${ }^{\otimes}$ Abstract published in Advance ACS Abstracts, January 1, 1996.

(1) Bénard, J., Ed. Adsorption on Metal Surfaces; Elsevier: New York 1983, and references therein.

(2) Hwang, R. Q.; Zeglinski, D. M.; López Vázquez-de-Parga, A.; Ogletree, D. F.; Somorjai, G. A.; Salmeron, M. Phys. Rev. B 1991, 44 , 1914.

(3) Ogletree, D. F.; Ocal, C.; Marchon, B.; Somorjai, G. A.; Salmeron, M.; Beebe, T. P.; Siekhaus, W. J. Vac. Sci. Technol. A 1990, 8, 297.

(4) Ogletree, D. F.; Hwang, R. Q.; Zeglinski, D. M.; López Vázquezde-Parga, A.; Somorjai, G. A.; Salmeron, M. J. Vac. Sci. Technol. B 1991, 9, 886 .

(5) Widring, C. A.; Alves, C. A.; Porter, M. D. J. Am. Chem. Soc. 1991, 113, 2805.

(6) Othani, H.; Kao, C. T.; Van Hove, M. A.; Somorjai, G. A. Prog. Surf. Sci. 1986, 23, 155

(7) Van Hove, M. A.; Wang, S. W.; Ogletree, D. F.; Somorjai, G. A. Quantum Chem. 1989, 20, 1.

(8) Maurice, V.; Marcus, P. Surf. Sci. 1992, 262, L 59.

(9) Zubimendi, J. L.; Salvarezza, R. C.; Vázquez, L.; Arvia, A. J. Langmuir 1996, 12, 2. such as argon and krypton on C(0001) also occurs at hollow sites of the substrate. ${ }^{1}$ The adsorption of sulfur atoms on the basal plane of graphite leads to $\mathrm{a} \sqrt{3} \times \sqrt{3}$ lattice with a sulfur - sulfur distance, $d_{S-S}=0.42 \mathrm{~nm}$ (Figure $\left.1 \mathrm{~A}\right)$, and hexagonal sulfur trimer adsorbates with $d_{S-S} \cong 0.24 \mathrm{~nm}$ (Figure 1B). These $d_{S-S}$ values differ from $d_{S-S} \cong 0.21 \mathrm{~nm}$ expected from bulk sulfur. This difference presumably reflects the substrate influence on the sulfur adsorbate structure as it occurs for adsorbate-adsorbate distances on surfaces of all types. ${ }^{1}$

Tunneling current $\left(I_{\mathrm{t}}\right)$ vs bias voltage $\left(V_{\mathrm{t}}\right)$ plots were obtained during constant current imaging by opening the feedback loop for a short time and measuring the tunneling current while the bias voltage was ramped. Each one of these plots (Figure 1C) exhibits different local density of states at the surface. Dashed curve exhibits a remarkable increase in tunneling current at sulfur adsorbate domains (Figure 1C, plot b) with respect to the bare graphite regions (Figure 1C, plot a). This fact disagrees with calculations made for sulfur atom adsorption on metals which predict that a sulfur atom adsorbed on a top metal atom position leads to an electron density minimum in STM images, ${ }^{10}$ in contrast to sulfur atom adsorption at hollow sites. This situation encourages a theoretical approach to sulfur atom adsorption on $\mathrm{C}(0001)$ for exploring the stability of possible sulfur adsorbate configurations. This information could lend a further support to the various adsorbate structures derived from STM imaging and explain their different behavior as compared to the sulfur-metal and S(gas)$\mathrm{C}(0001)$ system. ${ }^{1}$

For the purpose of this work quantum chemistry calculations are approached using the modified neglect of diatomic overlap (MNDO) Hamiltonian. The potential energy curves for sulfur atom adsorption on C(0001) clusters indicate that atop and bridge positions are favored with adsorption energies in the $0.3 \mathrm{eV} \leq E_{\mathrm{ad}} \leq 0.4 \mathrm{eV}$ range, whereas repulsive interactions are found at hollow sites. Data for adsorbates consisting of either a sulfur dimer or trimer on $\mathrm{C}(0001)$ clusters follow the trend observed for the adsorption of a single sulfur atom. These results support the structural analysis based on atomic resolution STM imaging data.

(10) Dounthy, J. C.; Ogletree, D. F.; Salmeron, M. C.; Sautet, P.; Bouquet, M. L.; Joachim, C. Ultramicroscopy 1992, 42-44, 490. 


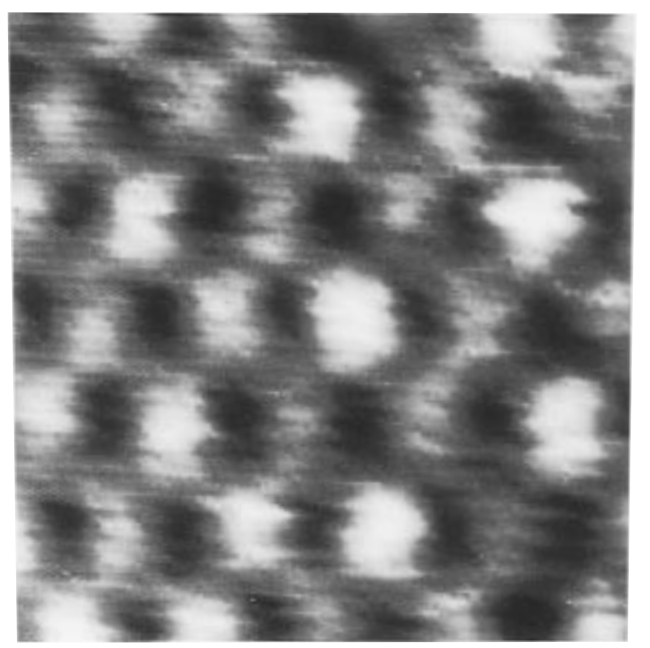

A

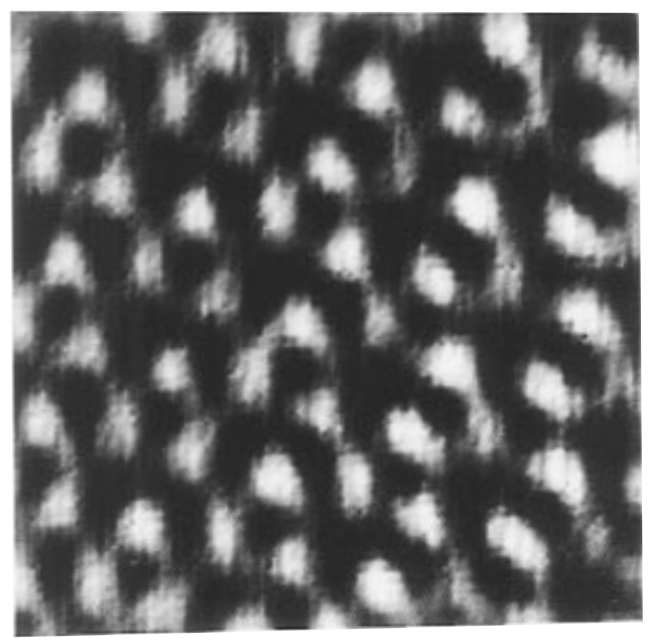

B

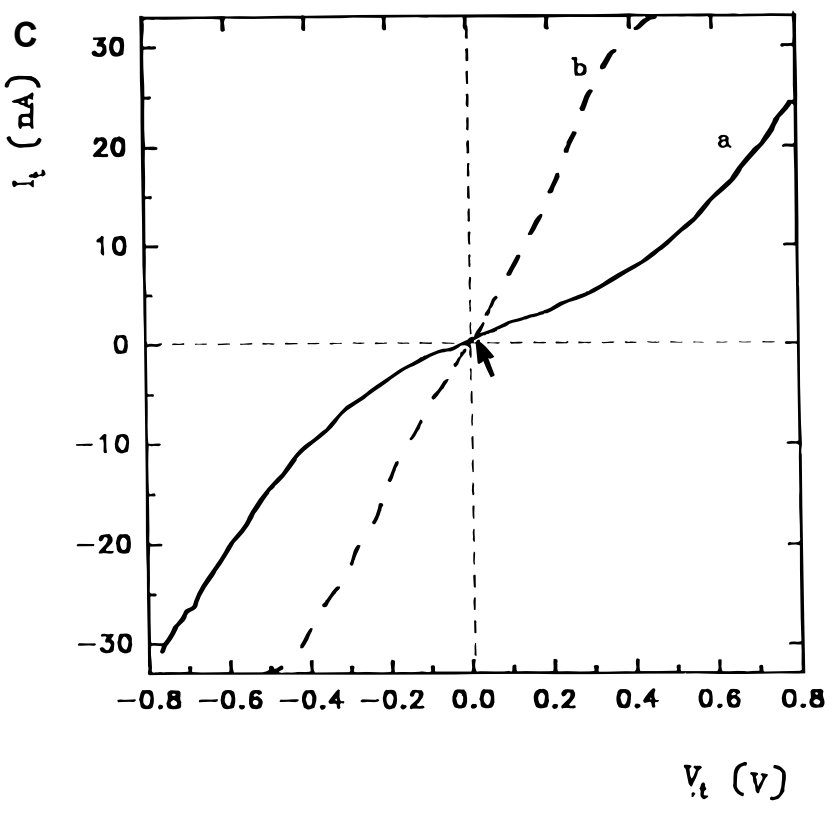

Figure 1. (A) Scanning tunneling microscopy image, constant height mode (top view), of a $\mathrm{C}(0001)$ surface covered with a $\sqrt{ } 3 \times \sqrt{ } 3$ sulfur lattice: tunneling current, $1 \mathrm{nA}$; bias voltage, $2.1 \mathrm{mV}$. (B) Scanning tunneling microscopy image, constant height mode (top view), of a C(0001) surface covered with a sulfur hexagonal trimer adsorbate: tunneling current, $1 \mathrm{nA}$; bias voltage, $2.1 \mathrm{mV}$. (C) Tunneling current vs bias voltage plot: tunneling current, $1 \mathrm{nA}$; bias voltage, $10 \mathrm{mV}$; (a) $\mathrm{C}(0001)$, blank; (b) sulfur adsorbate domain. The plot was recorded as indicated in the text. The arrow indicates the crossover at the bias voltage and tunneling current.

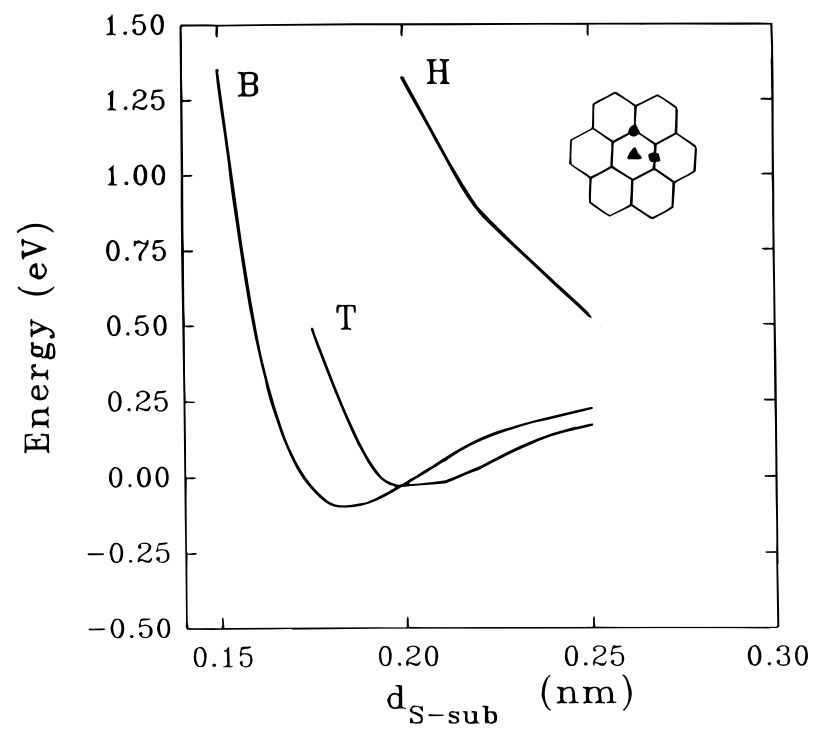

Figure 2. Potential energy curves for sulfur atom adsorption on "atop" ( $\mathrm{T}, \boldsymbol{\bullet})$, "bridge" (B, $\mathbf{\square})$, and "hollow" (H, $\mathbf{\Delta})$ sites.

\section{Calculation Procedure}

The theoretical method used to study the formation of sulfur adsorbates on the basal plane of graphite clusters was the MNDO Hamiltonian, using the MNDO QCMP113 Program provided by the Quantum Chemistry Program Exchange of the University of Indiana adapted to a PC486. The semiempirical molecular orbital method MNDO has been extensively tested and applied to a wide number of molecules, radicals, and triplet states of different type, yielding geometries (bond lengths and angles), ionization potentials, dipole moments, polarizabilities, heats of formation, and reaction mechanisms with accuracy. The original description of the MNDO method has been given by Dewar and Thiel. ${ }^{11 \mathrm{a}-\mathrm{c}}$ Parameters for atoms employed in our calculations were taken from those published by Dewar and Thiel and Dewar et al. ${ }^{11 a-c}$ The selection of this method was based on its efficiency when only s and p orbitals are involved, as is the case dealt with in this work. In addition, the success of the method in modeling surfaces within the cluster approach has been demonstrated, for example, in the case of Si surfaces both unreconstructed and reconstructed, ${ }^{11 \mathrm{~d}-\mathrm{g}}$ where its performance resulted comparable to ab initio and other semiempirical methods, in the reconstruction of $C(100)$ (diamond surface), ${ }^{11 \mathrm{~g}}$ and $C(111)$ surface ${ }^{11 \mathrm{~h}}$ which reconstructs due to a graphite-like bonding between the first two surface layers. Moreover, the MNDO method has been recently used to study the X-ray structure of coronene-potassium as a model for potassium (adsorbate) graphite surface interactions. ${ }^{11 \mathrm{i}}$

To model the $C(0001)$ substrate bidimensional honeycomb clusters of different sizes were employed, the largest cluster size (Figure 2) being determined by our computational capability. Dangling bonds were saturated with hydrogen atoms.

Firstly, the sulfur-substrate interaction energy of a single sulfur atom as a function of $d_{\mathrm{S}-\text { sub }}$, the sulfur atomsubstrate distance, was evaluated. The sulfur atom was

(11) (a) Dewar, M. J. S.; Thiel, W. J. Am. Chem. Soc. 1977, 99, 4899. (b) Dewar, M. J. S.; Thiel, W. J. Am. Chem. Soc. 1977, 99, 4907. (c) Dewar, M. J. S.; McKee, M. L.; Rzepa, H. S. J. Am. Chem. Soc. 1978, 100, 3607. (d) Lutrus, C. K.; Oshiro, T.; Hagen, D. E.; Sucksalk, S. H. Phys. Rev. B 1993, 48, 15086, and references therein. (e) Badziag, P.; Verwoerd, W. S. Phys. Rev. B 1989, 40, 1023. (f) Deak, P.; Snyder, L. C. Phys. Rev. B 1987, 36, 9612. (g) Verwoerd, W. S. Surf. Sci. 1983, 129, 419. (h) Gaigher, E.; Verwoerd, W. S. Surf. Sci. 1981, 103, 338. (i) Janiak, C.; Hemling, H. Chem. Ber. 1994, 127, 1251. 


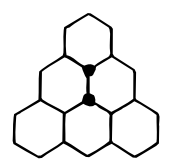

a)

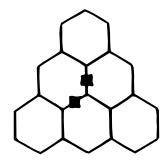

d)

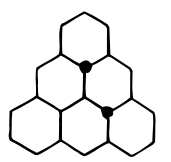

b)

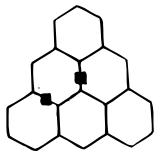

e)

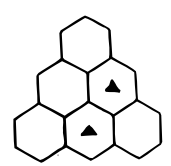

c)

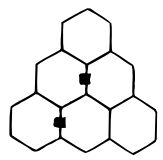

f)
Figure 3. Schemes of possible sulfur dimer adsorbates on the basal plane of a graphite cluster at sites of different symmetry.

placed in three different high symmetry positions, namely in "atop", "hollow", and "bridge" positions. Chemisorption of sulfur dimers and trimers was also studied at different substrate adsorption sites.

\section{Results}

The potential energy curve for a single sulfur atom on the basal plane of a graphite cluster is shown in Figure 2. Four different cluster sizes comprising from 3 to 8 carbon hexagons were used in the calculation. For all clusters the same dependence of the chemisorption energy on the sulfur atom-substrate distance $\left(d_{\mathrm{S}-\mathrm{sub}}\right)$ is found. Furthermore, it is found that atop and bridge site sulfur atom adsorptions on the substrate are the most favorable ones, unlike those in a hollow position.

Values $d_{\mathrm{S}-\text { sub }} \cong 0.20 \mathrm{~nm}$ and $d_{\mathrm{S}-\text { sub }} \cong 0.185 \mathrm{~nm}$, for a sulfur atom adsorbed in an atop and bridge position, respectively, were found. In both cases the adsorption of a sulfur atom on the basal plane of graphite involves an activation energy and, correspondingly, a limiting sulfurbasal plane of graphite bond energy. The reference energy level in our calculations corresponds to the substrate cluster surface atoms and sulfur atom at infinite distance from each other, leading to a chemisorption energy normalized per adsorbed sulfur atom. Values of $E_{\mathrm{ad}}$, the adsorption energies per adatom, derived from our calculations overall cluster sizes studied in this work, are in the range $0.3 \mathrm{eV} \leq E_{\mathrm{ad}} \leq 0.4 \mathrm{eV}$.

The adsorption energy for two sulfur atoms (dimer) adsorbed in atop, bridge and hollow positions (Figure 3), with the sulfur dimer axis parallel to the substrate plane was also evaluated. In this case, a chemisorption bond results only for a sulfur dimer in atop and bridge positions at the next-nearest neighbor lattice sites (Figure 4). Otherwise, no chemisorption results when the sulfur dimer is located in a hollow position (Figure 3c).

The adsorption of a sulfur dimer at a bridge site was also studied at three different values of $d_{S-S}$, the sulfur atom-sulfur atom distance (Figure $3 \mathrm{~d}-\mathrm{f}$ ). The corresponding potential energy curve exhibits a minimum binding energy value for $d_{\mathrm{S}-\mathrm{S}}=0.246 \mathrm{~nm}$ (Figure 4, bridge). No minima could be found for $d_{\mathrm{S}-\mathrm{S}}=0.146 \mathrm{~nm}$ (Figure 3d) and $d_{\mathrm{S}-\mathrm{S}}=0.213 \mathrm{~nm}$ (Figure $3 \mathrm{e}$ ). It should be noted that $d_{\mathrm{S}-\mathrm{S}}=0.18 \mathrm{~nm}$ results from the minimum binding energy of an isolated sulfur dimer.

The sulfur atom-substrate distance at the minimum binding energy (Figure 4 ) is nearly equal to that resulting from a single sulfur atom placed at the same site with $d_{\mathrm{S}-\mathrm{sub}}=0.185 \mathrm{~nm}$ for bridge binding, and $d_{\mathrm{S} \text {-sub }}=0.20 \mathrm{~nm}$ for atop binding (Figure 2).

Calculations were also done for a single sulfur trimer adsorbate on the basal plane of the graphite cluster in

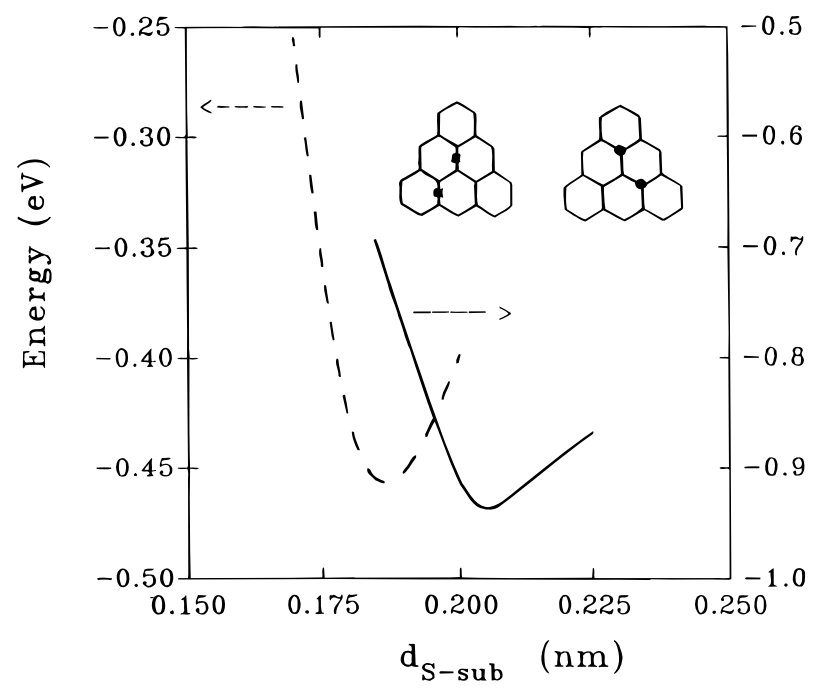

Figure 4. Potential energy curves for a sulfur dimer adsorbate on atop (solid line) and bridge (dashed line) site.

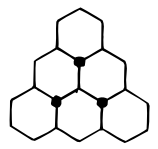

a)

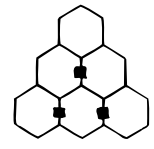

b)

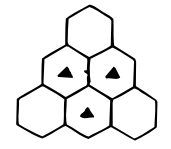

c)

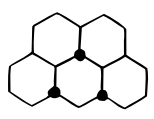

d)
Figure 5. Schemes of sulfur trimer adsorbates on sites of the basal plane of a graphite cluster of different symmetry.

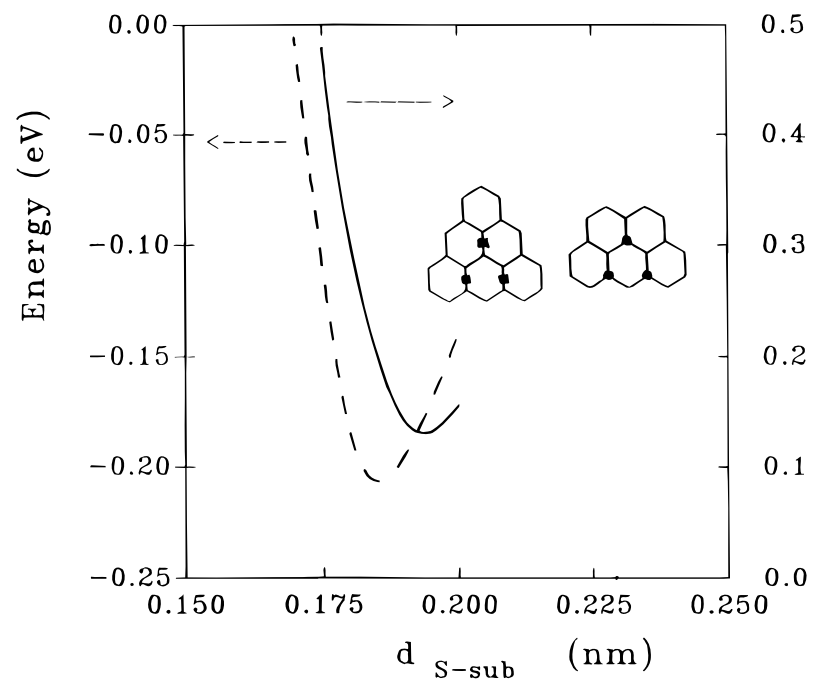

Figure 6. Potential energy curves for a sulfur trimer adsorbate on atop (solid line) and bridge (dashed line) sites.

atop, bridge, and hollow positions (Figure 5). In these cases minimum binding energy values appear only when the sulfur trimer is adsorbed in either bridge or atop position (Figure 6).

For dimer and trimer adsorbates in atop and bridge positions, the existence of a minimum in the potential energy curves indicates the possibility of stable adsorbate formation. Neither the evaluation of binding energies from the minimum at the potential energy curves nor the influence of dimer and trimer adsorbate-adsorbate indirect interactions on the sulfur-substrate binding energy can be made from these data, because of the size restriction imposed to the graphite clusters by our computational facilities.

The calculation procedure allowed us to evaluate the partial charge transfer value from the substrate to the adsorbed sulfur atom, which is approximately 0.35 


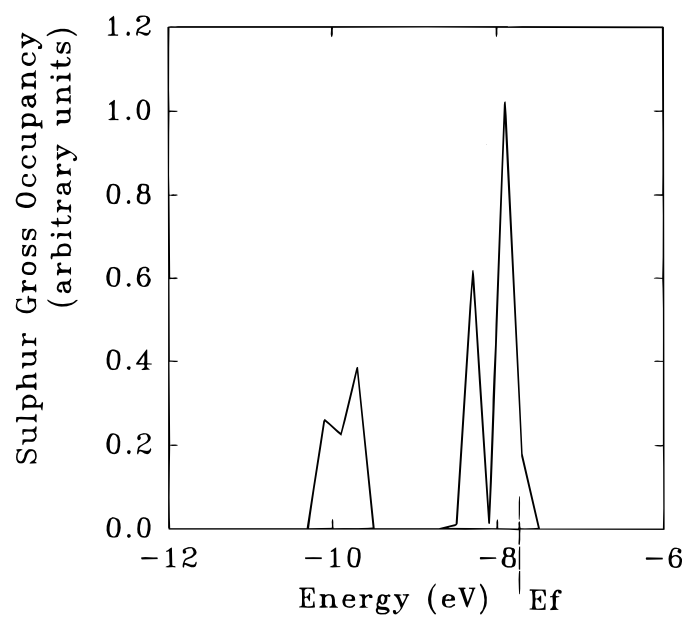

Figure 7. Sulfur density of states vs energy plot. The strong peak at the Fermi level corresponds to the resonance of $3 p$ sulfur orbitals.

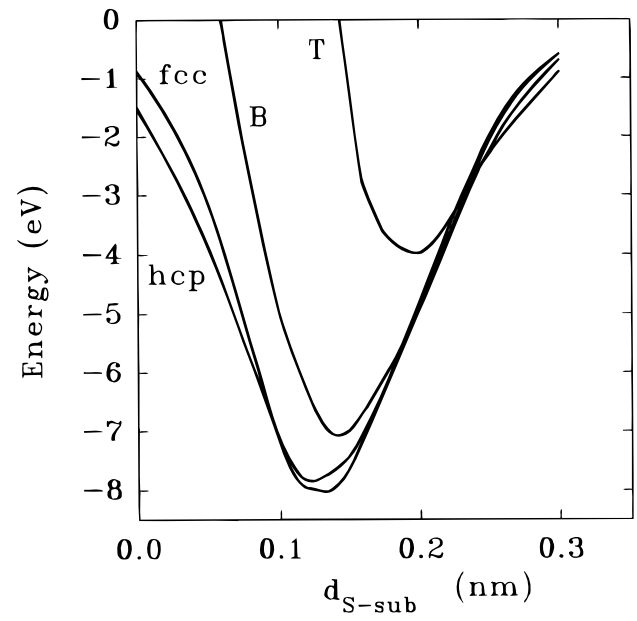

Figure 8. Potential energy vs sulfur atom-substrate distance for a sulfur adatom on $\mathrm{Al}(111)$ at hcp (and fcc) hollow, bridge (B) and atop (T) sites.

electron/sulfur atom. This figure is consistent with values reported for other metal adsorption processes on foreign substrates. ${ }^{12}$ Furthermore, calculations (Figure 7) also indicate a strong coupling between sulfur atom $3 p$ states and substrate wave functions at the Fermi level of the substrate.

The validity of the calculation procedure was checked by applying it to the adsorption of sulfur on aluminum single crystal surfaces. ${ }^{13-15}$ For these systems it is known that sulfur adsorption occurs preferentially at hollow sites. Potential energy curves resulting from MNDO calculations for a single adsorbed sulfur atom at atop, bridge, and hollow sites are shown in Figure 8. It is clear that in this case the formation of a sulfur adsorbate at a hollow site

(12) Kolb, D. M. In Advances in Electrochemistry and Electrochemical Engineering, Gerischer, H., Tobias, C. W., Eds.; John Wiley \& Sons: New York, 1978; p 161.

(13) Ganz, E.; Sattler, K.; Clarke, J. Surf. Sci. 1989, 219, 33-67.

(14) Maurice, V.; Marcus, P. Surf. Sci. 1992, 275, 65-74.

(15) Feibelman, P. J. Phys. Rev. 1994, B49, 14632. is largely favored (Figure 8) in agreement with already reported data. ${ }^{13-15}$

\section{Theoretical and STM Data Comparison}

Results presented in this work reveal that bridge and atop sulfur adsorbate positions on the basal plane of graphite are the most favorable ones for the formation of single sulfur atom (Figure 1A), dimer, and trimer adsorbates (Figure 1B). Furthermore, calculations show the likely formation of a $\mathrm{S}-\mathrm{C}$ bond for those adsorbates formed at atop and bridge sites, in contrast to repulsive interactions resulting from hollow sites.

Sulfur dimer and trimer adsorption in atop and bridge positions of the basal plane of graphite imply $d_{S-S} \cong 0.246$ $\mathrm{nm}$. This distance coincides with that resulting from the hexagonal trimer sulfur structures imaged by STM (Figure 1B). In fact, it also reflects the influence of the substrate on sulfur adsorption.

On the other hand, the strong coupling between the adsorbed sulfur atom 3p-states and substrate orbitals at the Fermi level, and the relevant charge transfer contribution to adsorption binding energy can explain the remarkable increase in the tunneling current. This increment is quite noticeable in the tunneling current vs bias voltage plot (Figure 1C) when the STM tip goes over a domain of the graphite surface which is covered by sulfur atoms.

\section{Conclusions}

Results from the application of the MNDO method to the evaluation of the adsorption energy of sulfur atoms on the basal plane of graphite clusters can be summarized as follows.

(i) Adsorbed sulfur atom formation in atop and bridge substrate positions is favored. The corresponding binding energy is in the $0.3 \mathrm{eV} \leq E_{\mathrm{ad}} \leq 0.4 \mathrm{eV}$ range. Similar configurations may appear for sulfur dimer and trimer adsorbates.

(ii) Single sulfur atom and sulfur dimer or trimer adsorption bonding to hollow positions on the basal plane of graphite leads to unstable adsorbate configurations.

(iii) Values $d_{\mathrm{S}-\text { sub }}=0.185 \mathrm{~nm}$ and $d_{\mathrm{S}-\text { sub }}=0.20 \mathrm{~nm}$ at bridge and atop sites, respectively, were found for a single sulfur atom, dimer, and trimer adsorbates.

(iv) A partial charge transfer from the substrate to the adsorbed sulfur atom which was estimated as 0.35 electron per sulfur atom, contributes to the adsorption binding.

(v) The strong coupling between sulfur atom $3 p$ state and substrate orbitals at the Fermi level causes a considerable increase in the density of states near the Fermi level. This fact can explain the remarkable increase in the tunneling current when the tip scans over sulfurcovered substrate domains.

Acknowledgment. This work was financially supported by the Consejo Nacional de Investigaciones Científicas y Técnicas of Argentina (CONICET), and by the scientific cooperation agreement between CONICET and CSIC, Consejo Superior de Investigaciones Científicas of Spain. J.L.V. is a member of the Research Career of CIC (Bs As).

\section{LA940688R}

